\title{
ESSAI DE CLASSIFICATION DES CÉNURES (1)
}

\author{
Par Juan J. ANGULo et Agustin L. ROQUE
}

L'examen des travaux publiés montre que les cénures peuvent présenter plusieurs types de développement. En effet, on a trouvé dans une seule cavité de nombreux cénures de forme régulière et aussi de forme irrégulière. D'autre part, Henry et Ciuca (1914), Railliet et Marullaz (1919), Schwartz (1927), Coutelen (1944), Angulo et Roque (1948) ont rapporté des cas dans lesquels des cénures de forme irrégulière se sont développés dans des membranes réactionnelles alvéolaires, en rayon de miel. On sait peu de chose sur le développement des cénures et sur les causes de leur polymorphisme. Il a été entrepris peu d'expériences pour résoudre ce problème et il ne semble pas qu'on ait essayé d'établir une classification des cénures.

Une larve voisine, l'hydatide, a été étudiée à fond et la classification de ses types de développement (Dévé, 1912) a été généralement acceptée, quoiqu'avec des modifications de nomenclature. Dévé (1916) et Dew (1925) ont étudié expérimentalement le développement de quelques types d'hydatides, et étudié aussi des hydatides développées spontanément (Dévé, 1912 et 1918 ; Dew, 1925). Ils ont conclu que le développement des hydatides peut être influencé par plusieurs facteurs, dont la révision est donnée ci-dessous.

Dans ce travail, nous proposons une classification des cénures suivant leurs types de développement et nous passons en revue les facteurs qui peuvent provoquer l'apparition de ces divers types.

\section{Classification proposée}

Deux spécimens de cénures ont été étudiés : Multiceps serialis (1944) et Multiceps sp. (1948). Tous deux ont été rencontrés chez Capromys pilorides. Multiceps serialis était nettement diverticulé, mais s'était développé dans une cavité simple, de forme régulière,

(1) Traduit de l'anglais par le $\mathrm{D}^{r}$ Maurice Langeron.

Ans. de Parasitologie, T. XXIV, Nos $3-4$. - 1949, p. 200-206. 
recouverte par une membrane réactionnelle lisse. Multiceps sp., bien que de même largement diverticulé, s'était développé dans une membrane réactionnelle irrégulière, alvéolaire. Beaucoup de descriptions analogues et des cas de cénures non diverticulés existent dans la littérature. Comme il y a des variations dans la diverticulation des cénures et dans la structure des membranes réactionnelles correspondantes, il apparaît que les cénures peuvent être répartis en plusieurs types.

Des cénures de forme régulière, sans diverticules, situés, chez l'hôte, dans des cavités simples, ont été observés par Numan (in Hall, 1910, Kunsemüller (1903), Moussu et Dallar (in Hall, 1910), Hall (1910), Henry et Ciuca (1914), Blacklock et Southwell (1935) et par Coutelen (1944).

Des cénures de forme irrégulière, diverticulés, mais développés dans des cavités simples de l'hôte, ont été décrits par Kunsemüller (1903), Henry et Ciuca (1914), Bonnal, Joyeux et Bosch (1933), Brumpt, Duvoir et Sainton (1934), Cannon (1942), Roger, Sautet et Paillas (1942), Coutelen (1944) et Kouri et Angulo (1944).

Les vésicules non diverticulées peuvent donc être réunies sous le nom de cénures réguliers. uniloculaires et les cénures diverticulés peuvent être désignés par le nom de cénures irréguliers uniloculaires. Le type de structure des cénures décrits par Henry et Ciuca (1914), Railliet et Marullaz (1919), Schwartz (1927), Coutelen (1944) et par Angulo et Roque (1948), montre qu'il existe un autre type de développement, celui des cénures multiloculaires.

Cette classification se trouve confirmée par une comparaison entre les cénures et les hydatides. Les cénures uniloculaires et les hydatides sont évidemment semblables. Les cénures et les hydatides multiloculaires présentent des caractères communs importants : membrane réactionnelle alvéolaire, abondance de vésicules stériles et petite taille de ces vésicules. Les cénures et les hydatides sont les seules larves de cestodes renfermant plusieurs scolex dans une seule vésicule et formant des vésicules filles.

Une étude minutieuse des descriptions existant dans la littérature démontre la nécessité de reconnaître deux sous-types de cénures uniloculaires. Le cénure régulier uniloculaire paraît caractéristique des cénures développés dans le cerveau, tandis que les formes irrégulières uniloculaires et les cénures multiloculaires paraissent se développer dans le tissu conjonctif. La présence simultanée de ces deux types, uniloculaire et multiloculaire, dans le même cénure (Railliet et Marullaz, 1919 ; Coutelen, 1944), indique qu'aucun de ces deux types de structure n'est un caractère spécifique. Cette hypo- 
thèse est appuyée par des constatations analogues (Dévé, 1912 ; Dew, 1926) chez des hydatides de bovins et de moutons. Comme la membrane réactionnelle est la réponse de l'hôte à la présence du
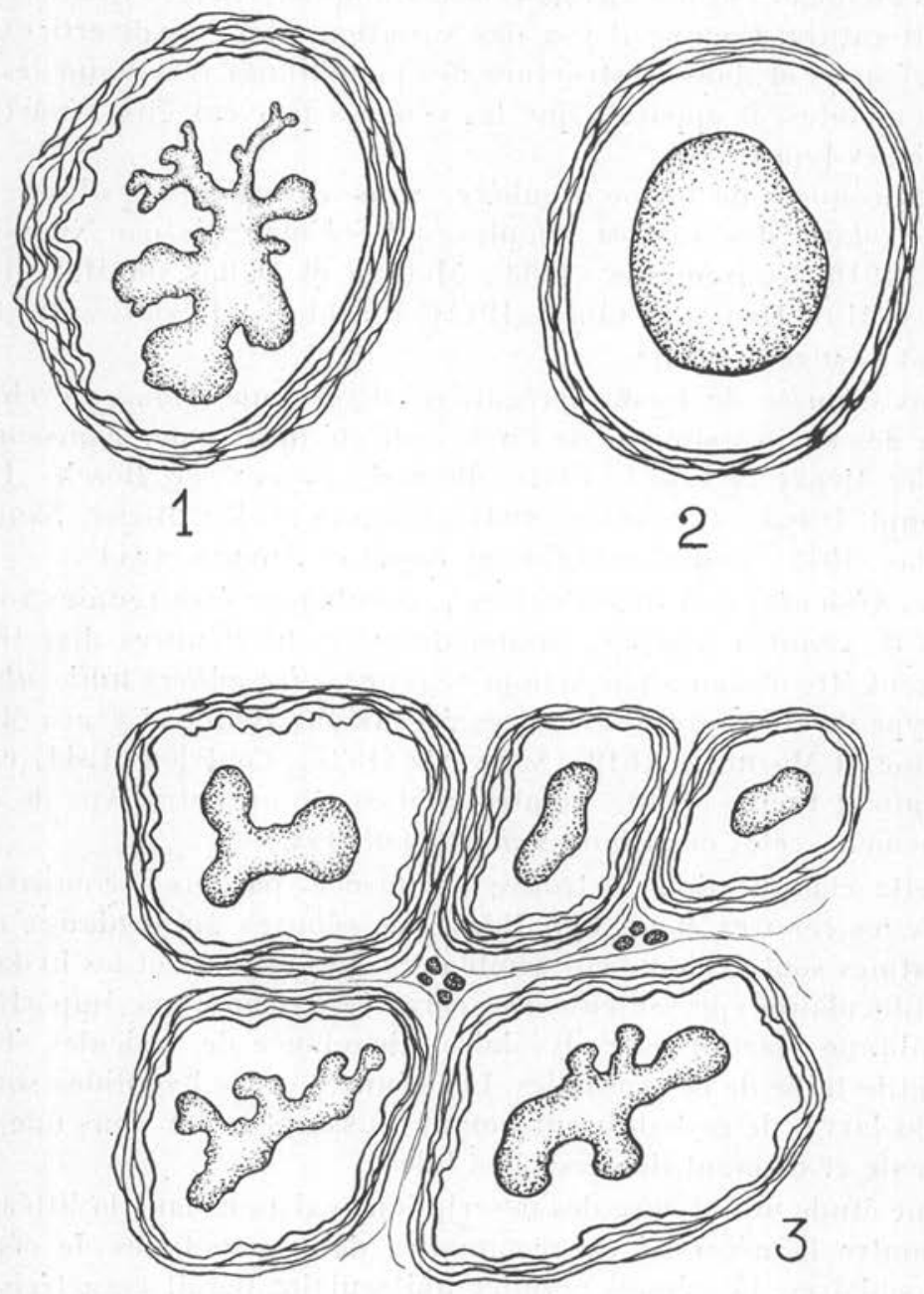

Fı. - 1, Cénure uniloculaire irrégulier ; 2, cénure uniloculaire régulier ; 3 , cénure multiloculaire.

parasite, il est évident que la membrane réactionnelle réticulée du cénure inultiloculaire dépend d'un type larvaire défini.

La figure rend compte de cette classification des cénures. Les définitions des types de cénures proposés sont les suivantes: 
I. Cénure uniloculaire. - C'est la larve des espèces du genre Multiceps Goeze 1782, développée dans une cavité unique. La membrane réactionnelle, ou le tissu de l'hôte légèrement modifié, est périphérique el peut présenter une diverticulation. On reconnait deux sous-types :

A. Cénure uniloculaire régulier. - Vésicule de forme régulière, avec diverticules rares ou peu visibles.

B. Cénure uniloculaire irrégulier. - Vésicule de forme irrégulière, résultant de la présence de vésicules filles, avec diverticules et scolex évaginés.

II. CÉnure multiloculaine. - Larve des espèces du genre Multiceps Goeze 1782, formée de nombreuses vésicules développées à l'intérieur des cavités d'une membrane réactionnelle en gâteau de miel. Dans ce type, les vésicules sont petites et il y en a souvent de stériles.

La principale différence pratique entre les cénures uniloculaires et multiloculaires est que les premiers peuvent être isolés en entier du tissu de l'hôte, ce qui n'est pas possible pour les autres. Des vésicules de type différent peuvent coexister étroitement, mais ce fait n'est pas en contradiction avec la classification ci-dessus. La distinction de ces types de cénures des divers types d'hydatides et des cysticerques, cysticercoïdes, etc..., se fait au moyen des caractères suivants, pris en totalité ou séparément : histologie de la paroi de la vésicule, lieu d'origine des scolex, présence de diverticules et de vésicules filles, nombre des scolex par vésicule, tissu dans lequel la larve se développe.

De notre définition du cénure multiloculaire, on peut conclure que le cénure dénommé multiloculaire par Cobbold (1861) n’était pas en réalité, multiloculaire, parce qu'il n'y a pas de preuve, dans sa description ultérieure (1864), qu'il se trouvait dans une membrane réactionnelle en gâteau de miel. La larve de Cobbold doit donc être considérée comme un cénure uniloculaire irrégulier.

Dew (1926) a proposé une classification des hydatides, mais a fait un mauvais usage des termes scientifiques en les appliquant aux types morphologiques de cénures et à la spécification de l'hôte. Il a employé aussi une nomenclature (kyste simple univésiculaire, kyste multiloculaire et masses polykystiques) qui augmente la confusion déjà existante dans la nomenclature des hydatides. De plus, la membrane réactionnelle n'est pas prise en considération dans cette classification.

\section{Discussion DEs FACTEurs de Croissance}

- D'après Henry et Ciuca (1914), on observe des vésicules filles dans le cénure de Multiceps serialis au bout de six à huit mois, aussi 
eroyaient-ils que le type multiloculaire résulte de l'âge. Les cénures de Multiceps serialis décrits par Bonnal, Joyeux et Bosch (1933), par Brumpt, Duvoir et Sainton (1934) et par Kouri et Angulo (1944) atteignaient une grande dimension, portaient beaucoup de scolex bien développés, mais ne montraient pas de partition entre les vésicules filles. Au contraire, le cénure trouvé par Angulo et Roque (1948) était petit, les scolex étaient peu nombreux et incomplètement développés, mais il était multiloculaire. Il apparaît donc que l'àge de la larve n'est pas un facteur décisif dans le développement des cénures multiloculaires. Henry et Ciuca (1914) rapportent aussi que lorsque plusieurs scolex se développent en même temps enkyştés, le cénure primitif ne peut être distingué des cénures secondaires.

Coutelen (1944) croyait que la morphologie irrégulière de quelques cénures de $M$. serialis était due à des facteurs locaux mécaniques, tels que des tractus fibreux, des aponévroses, des tendons, de gaines dis nerfs ou de vaisseaux sanguins, etc..., et aussi à la traction et aux pressions soudaines, fortes et successives, exercées sur le cénure par les contractions musculaires de l'hôte, le tout agissant comme obstacle au développement du cénure. Il insiste sur le fait que les cénures développés librement dans les cavités séreuses ont une forme régulière, tandis que ceux des tissus conjonctifs sous-cutané ou intramusculaire présentent de nombreux diverticules. Comme cause possible de la morphologie irrégulière des larves de $M$. serialis, Coutelen (1944) signale les irrégularités de la membrane réactionnelle et aussi sa cuticule mince et délicate, par comparaison avec la cuticule épaisse, résistante et lamelleuse de l'hydatide. Un cénure de $M$. serialis de forme irrégulière (Kouri et Angulo 1944) a acquis une grande taille à l'intérieur d'une membrane réactionnelle régulière, kystique, dont la surface interne était très lisse, sans la moindre poche. Clapham (1942) soutenait que $M$. serialis et $M$. multiceps étaient la même espèce. Les cénures réguliers de $M$. multiceps, qu'on trouve dans le cerveau des moutons, ont une cuticule mince et délicate, mais ne sont pas polymorphes. Aussi ne sommes-nous pas de l'avis de Coutelen en ce qui concerne l'importance des irrégularités et de la minceur de la membrane réactionnelle, comme cause de la forme irrégulière de quelques cénures.

Suivant Dévé (1912 et 1916), les kystes hydatiques multiloculaires sont produits par la formation de vésicules filles exogènes. Dew (1925) prétend que les hydatides multiloculaires sont souvent dues à une croissance le long de lignes de moindre résistance, plutôt qu'à. un bourgeonnement continu. Dévé (1918) et Dew (1925) croyaient 
que la formation des vésicules filles dans lẹs kystes hydatiques uniloculaires résulte d'une interférence avec son développement normal et, par conséquent, qu'elle peut être considérée comme un mécanisme de défense en vue de la reproduction. Dévé (1912) croyait que l'hydatide, dite multiloculaire, des os n'est pas une forme définitive, mais simplement un artifice morphologique provoqué par la structure osseuse alvéolaire sur un parasite plastique. Dévé (1916) considérait que la formation de vésicules filles intracuticulaires dans une hydatide osseuse est due à un mécanisme de défense en vue de la reproduction.

\section{ReMERCIEMENTS}

Les auteurs remercient le $\mathrm{D}^{r}$ Irving Rappoport, du Collège médical de l'Université Cornell, pour son assistance dans la préparation du manuscrit, et le $\mathrm{D}^{\mathrm{r}}$ Pédro Kouri, de l'Institut de médecine tropicale de l'Université de la Havane, pour les facilités qu'il nous a accordées dans son laboratoire.

\section{RÉSUMÉ}

Une classification est proposée pour les cénures, suivant leurs types de structure ; elle est basée sur l'étude de deux cas, ainsi que sur les descriptions publiées par d'autres auteurs. Les deux types proposés sont le type uniloculaire et le type multiloculaire. Le cénure uniloculaire est divisé en outre en deux sous-types : régulier et irrégulier. Ces types de structure n'ont probablement aucun rapport avec les espèces. Les facteurs qui gouvernent ces types sont passés en revue et discutés.

\section{Bibliographie}

Axgulo (J. J.) et RoQue (A. L.). - J. Parasit., XXXIV, 1948.

Blacklock (D.) et Southwel. (T.). - A Guide to Human Parasitology, Baltimore, William Wood Co, 1935.

Bonnal (G.), Joyeux (C.) et Bosch (P.). - Bull. Soc. Path. Exot., XXVI, 1933, 1060.

Brumpt (E.), Duvorr (M. E.) et Santon (J.). - Ann. Parasit., XII, 1934, 371.

Cannon (D. A.). - Ann. Trop. Med. Parasit., XXXVI, 1942, 32.

Clapham (P. A.). - J. Helminth., XX, 1942, 31.

Сoвbold (T. S.). - Proc. Zool. Soc. London, 1861, 117.

- Entozoa: An Introduction to the Study of Helminthology, London, Groombridge and Sons, 1864.

Coutelen (F.). - C.R. Soc. Biol. Paris, CXXXviII, 1944, 104. 
DÉvÉ (F.). - Premier Congrès international de Pathologie Comparée, 1912, 363.

- Arch. Méd. Exp., XXVIII, 1916, 113, 301 et 357.

- Presse Méd., XXVI, 1918, 413.

DEw (H. R.). - Med. J. Australia, XI, 1925, 101, 497.

- Med. J. Australia, XII, 1926, 301.

Hall (M. C.), - U.S. Dpt. Agric., Bureau Anim. Ind., Circular 165, 1910.

Henry (A.) et Ciuca (A.). - Ann. Inst. Pasteur, XXVIII, 1914, 365.

Kouni (P.) et Angulo (J. J.). -- Rev. Med. Trop. Parasit., La Havane, X, 1944, 64. Iunsemüller (F.). - Zool. Jahrb., Abth. Anat. Ontog. Thiere, XVIII, 1903, 507. Railliet (A.) et Marullaz (M.). - Bull. Soc. Path. Exot., XII, 1919, 223.

Roger (H.), Sautet (J.) et Paillas (J. E.). - Rev. Neurol., LXXIV, 1942, 319. Schwartz (B.). - J. Agric. Res., XXXV, 1927, 471.

Université de la Havane, Cuba, Ecole de Médecine. Départements de pathologie expérimentale et d'histologie. 